\title{
artigo
}

\section{Perfil de saúde da tuberculose entre crianças e adolescentes indígenas: uma revisão integrativa}

Tuberculosis health profile in indigenous children and adolescents: an integrative review

Perfil de salud de la tuberculosis em niños y adolescentes indígenas: uma revisión integradora

\begin{abstract}
RESUMO
Objetivo: Analisar como a literatura tem abordado o perfil de saúde da tuberculose entre crianças e adolescentes indígenas. Método: Revisão integrativa realizada com descritores indexados nas bases de dados Medline, Scopus, Embase, Web of Science e Lilacs a partir de estudos primários, com textos completos, publicados no período de 2000 a 2020 . Resultados: Foram encontradas 726 publicações e após a aplicação dos critérios previamente estabelecidos, 10 artigos foram considerados elegíveis, realizados exclusivamente no Brasil, entre 2004 e 2019, maior incidência em menores de 5 anos, predomínio do sexo masculino, forma clínica pulmonar, sendo o exame de radiografia de tórax a variável clínica mais explorada nos estudos, baixa realização do teste tuberculínico e avaliação nutricional, além de elevada taxa de cura. Conclusão: Evidencia-se a dificuldade diagnóstica, incluindo baixa realização dos exames, não sistematização da busca dos achados clínicos, ausência de avaliação nutricional e investigação de contatos.
\end{abstract}

DESCRITORES: Perfil de saúde; Tuberculose; Povos Indígenas; Criança; Adolescente.

\section{ABSTRACT}

Objective: To analyze how the literature has addressed the tuberculosis health among indigenous children and adolescents. Method: An integrative review conducted with descriptors indexed in the Medline, Scopus, Embase, Web of Science, and Lilacs databases from primary studies, with complete texts, published in the period 2000 to 2020. Results: 726 publications were identified, and after the application of the previously established criteria, 10 articles were considered eligible, carried out exclusively in Brazil, between 2004 and 2019, with a higher incidence in children under 5 years old, a predominance of the male gender, clinical pulmonary form, being the chest radiography the clinical variable most explored in the studies, the low performance of the tuberculin skin test and nutritional assessment, in addition to a high cure rate. Conclusion: Diagnostic difficulties are evident, including the low exam performance, failure to systematize the search for clinical findings, absence of nutritional assessment, and investigation of contacts.

DESCRIPTORS: Health Profile; Tuberculosis; Indigenous Peoples; Child; Adolescent.

\section{RESUMEN}

Objetivo: Analizar cómo la literatura ha abordado el perfil de salud de la tuberculosis en niños y adolescentes indígenas. Método: Revisión integradora realizada con descriptores indexados en las bases de datos de estúdios primarios Medline, Scopus, Embase, Web of Science y Lilacs, com textos completos, publicadas en el período 2000 y 2020. Resultados: Se encontraron 726 publicaciones y, previa aplicación de criterios estabelecido, se consideron elegibles 10 artículos, realizados exclusivamente en Brasil, de 2004 y 2019, con mayor incidencia en menores de 5 años, predominantemente varones, uma forma clínica pulmonar, siendo la radiografia de tórax la variable clinica más explorada em los estúdios, puebra cutânea de tuberculina baja y evaluación nutricional, además de una alta tasa de curación Conclusión: Las dificultades diagnósticas son evidentes, incluyendo mala realización de exámenes, falta de sistematización de la búsqueda de hallazgos clínicos, ausência de valoración nutricional e investigación de contatos.

DESCRIPTORES: Perfil de Salud; Tuberculosis; Pueblos Indígenas; Niño; Adolescente.

RECEBIDO EM: 28/01/2021 APROVADO EM: 09/02/2021

\section{Gisele Aparecida Soares Cunha de Souza}

Graduanda em Medicina pela Universidade Federal de Rondônia (UNIR), Porto Velho-RO.

ORCID: 0000-0001-7226-4476 


\section{Melisane Regina Lima Ferreira}

Enfermeira, Especialista em Saúde Pública, Doutoranda pela Escola de Enfermagem de Ribeirão Preto, Universidade de São Paulo (EERP-USP), Ribeirão Preto- SP.

ORCID: 0000-0003-1694-5124

\section{Rafaele Oliveira Bonfim}

Enfermeira, Mestre em Ciências pela Escola de Enfermagem de Ribeirão Preto, Universidade de São Paulo (EERP-USP), Ribeirão Preto- SP.

ORCID: 0000-0001-8157-2323

\section{Nathalia Halax Orfão}

Enfermeira, Doutora em Ciências, Docente no Departamento de Medicina da Universidade Federal de Rondônia (UNIR), Porto Velho-RO.

ORCID: 0000-0002-8734-3393

\section{INTRODUÇÃO}

A tuberculose (TB) é uma doença infectocontagiosa, causada pelo Mycobacterium tuberculosis, que atinge todos os sexos, faixas etárias e etnias, contudo, de forma heterogênea. Está entre as 10 principais causas de óbito no mundo e, em 2019, 10 milhões de casos novos de TB foram notificados e 1,2 milhões foram a óbito pela doença ${ }^{(1)}$.

Populações vulnerabilizadas e com maior risco de adoecimento pela $\mathrm{TB}$, tais como as pessoas vivendo com o vírus da imunodeficiência adquirida (HIV), privada de liberdade, profissionais da saúde, em situação de rua e os povos indígenas ${ }^{(2)}$, são consideradas prioritárias para a realização de estratégias e ações de vigilância em saúde para o controle da doença ${ }^{(3)}$.

Os indígenas são povos minoritários, representam $5 \%$ da população mundial e se distribuem em 90 países. O cuidado de saúde desses povos precisa ser diferenciado, uma vez que apresentam peculiaridades étnicas e culturais ${ }^{(3)}$. Cada país possui programas específicos para o cuidado da saúde indígena tal como, na Austrália, por meio do Programa de Saúde dos Australianos Indígenas (IAHP), o qual é responsável pelo acesso ao Serviço de Saúde Primário $^{(4)}$; no Canadá, através dos programas Federais, Provinciais e Municipais ${ }^{(5)}$ e no Brasil, com um subsistema conhecido como lei Arouca, criado em 1999, no Sistema Único de Saúde (SUS) ${ }^{(6)}$.

Os dados relativos à $\mathrm{TB}$ entre os povos indígenas nos diversos países sofrem alta variabilidade por representarem diferentes etnias, faixas etárias, regiões e amostragens. Para compreender e acompanhar as necessidades de saúde desses povos, é fundamental a criação de relatórios de saúde unificados, os quais permitiriam abranger o estado de saúde desta população, de forma a compreender como tem sido desenvolvidas as ações específicas para o controle da doença, comparar e mensurar os indicadores operacionais e epidemiológicos, tal como é realizado para a TB no Global Tuberculosis Report ${ }^{(1)}$.

No Brasil, a TB nas populações indígenas é uma doença preocupante, cujo risco de adoecimento é o triplo quando comparado com a população em geral $^{(2)}$, que em alguns casos, dependendo da região e do ano avaliado, chega a ser 10 vezes maior ${ }^{(7)}$. Aliado a isso, soma-se as desigualdades sociais relacionadas às barreiras geográficas, linguísticas e culturais entre os povos, além das dificuldades de acesso ao SUS, mesmo o país possuindo um subsistema específico para atenção à saúde dos povos indígenas ${ }^{(8,9)}$.

Ademais, quando se restringe a análise de saúde para a população infanto-juvenil indígena, o risco de adoecimento aumenta significativamente, uma vez que apresentam sintomas que se assemelham a outras patologias características da idade, com isso, a realização da coleta de escarro e a baixa quantidade de bacilos nas lesões, dificultam o diagnóstico para a $\mathrm{TB}^{(2)}$. Ainda, as revisões de literatura sobre TB indígena em crianças e adolescentes são escassas e estão focadas no diagnóstico para TB latente e séries de casos de TB extrapulmonar ${ }^{(10,11)}$. Neste sentido, questiona-se qual o perfil sociodemográfico, epidemiológico e clínico desse recorte populacional?

Assim, este estudo teve como objetivo analisar como a literatura tem abordado o perfil de saúde da TB entre crianças e adolescentes indígenas.

\section{MÉTODOS}

Trata-se de uma revisão integrativa da literatura, desenvolvida em seis etapas, a saber: formulação da pergunta norteadora, estabelecimento dos critérios de inclusão e exclusão, localização dos estudos nas bases de dados, avaliação crítica do estudo para a seleção de publicações elegíveis, extração dos dados e análise, interpretação e síntese dos resultados encontrados ${ }^{(12)}$.

A partir da pergunta norteadora "Como a literatura científica tem abordado o perfil da TB entre crianças e adolescentes indígenas?", foi definido a estratégia PICo, no qual P (população) correspondeu aos povos indígenas, I (interesse) à TB e $\mathrm{Co}$ (contexto) relacionou-se às crianças e adolescentes.

Como critérios de inclusão, utilizou-se artigos primários, com textos completos, publicados no período entre 2000 a 2020, nos idiomas português, inglês e espanhol. E como exclusão, estudos duplicados, revisões, editoriais, estudos com abordagem não indígena, adultos, estudos fora do tema proposto e estudos de caso, o qual tinham como propósito avaliar as formas 
raras de TB, que não faziam parte do escopo dessa revisão.

Para a expressão de busca, utilizou-se o vocabulário livre e controlado, compostos pelos termos indexados nos Descritores em Ciências da Saúde (DeCS), Medical Subject Headings (MESH) e Embase Subject Headings (Emtree), com seus respectivos sinônimos em português, inglês e espanhol, combinados pelos booleano AND, além da utilização da técnica de trucagem dos termos nas bases de dados que permitiam, a fim de facilitar a pesquisa (Quadro 1).

A busca na literatura ocorreu no dia 14 de agosto de 2020, via portal de periódicos CAPES, por meio de acesso remoto, nas bases de dados Literatura latino Americana e do Caribe em Ciências da Saúde (LILACS), Medical Literatury Analysis and retrieval System Online/ PubMed (MEDLINE), EMBASE (Elsevier), SCOPUS (Elsevier) e Web Of Science (Elsevier). Considerou-se como campo de busca o título, resumo e palavras-chave.

Posteriormente, as publicações foram exportadas para o gerenciador de referências online Rayyan QCRI da Qatar Computing Research Institute ${ }^{(13)}$ para seleção dos estudos, por meio da leitura de título e resumo, por duas pesquisadoras independentes, cujas discordâncias foram resolvidas por uma terceira. As concordâncias foram mensuradas por meio do coeficiente Cohen Kappa, e obteve-se o valor 0,93 , considerado como concordância perfeita ${ }^{(14)}$.
Os artigos

selecionados

seguiram para

a etapa de leitura

na íntegra

e a confirmação da

elegibilidade

sobre o perfil

de saúde

ocorreu com

aqueles que

atendiam à

pergunta

norteadora.
Os artigos selecionados seguiram para a etapa de leitura na íntegra e a confirmação da elegibilidade sobre o perfil de saúde ocorreu com aqueles que atendiam à pergunta norteadora. Posteriormente, para a extração e análise dos dados, foi elaborada uma matriz de síntese com autor, ano, população, objetivo, delineamento e cenário do estudo, população, além dos principais resultados sociodemográficos, epidemiológicos e clínicos.

\section{RESULTADOS}

Foram encontradas 726 publicações nas bases de dados e, visando atender aos critérios previamente estabelecidos, 715 foram excluídas, das quais 201 por duplicação, 130 por estarem anteriores ao ano 2000, 22 por estarem em outros idiomas, seis por serem textos incompletos, além de 16 artigos de revisões de literatura, quatro estudos de caso e três editoriais (Figura 2).

Após a leitura do título e resumo, foram excluídos 93 artigos por não abordarem a população indígena, 72 pelo recorte voltado à população indígena adulta e 168 por discutirem outros temas como vacinação, nutrição, programas de saúde, informações geográficas, parâmetros hematológicos e educação em saúde. Dessa forma, 11 artigos foram considerados elegíveis para leitura na íntegra, dos quais apenas um foi excluído por que os resultados relativos aos indígenas e não indígenas foram apre-

\section{Quadro 1. Expressões de busca utilizada nas bases de dados para a revisão integrativa da literatura, Porto Velho,} Rondônia, Brasil, 2020.

\begin{tabular}{|l|l|}
\hline PICO & VOCABULÁRIO CONTROLADO E/OU LIVRE \\
\hline População: Indígenas & $\begin{array}{l}\text { "Indigenous Peopl**" OR "Indigenous Communities" OR "Pueblos Indígenas" OR "Etnia Nivaclé" OR } \\
\text { "Indígena Guarani" OR "Indígenas de Pykasú" OR "Personas Indígenas" OR "Pueblo Guarani" OR } \\
\text { "Pueblo Tupi" OR "Indio Guaraní" OR "Population Group*" OR "Indigenous Population*" OR "Tribes" }\end{array}$ \\
\hline & AND \\
\hline Interesse: Tuberculose & $\begin{array}{l}\text { Tuberculosis OR "Mycobacterium tuberculosis Infection*" OR "Koch* Disease" OR Tuberculose* } \\
\text { OR "Infección por Mycobacterium tuberculosis" OR "Bacillus tuberculosis" OR "Mycobacteria tu- } \\
\text { berculosis" OR TB }\end{array}$ \\
\hline Contexto: Criança e Adolescente & $\begin{array}{l}\text { Child* OR paediatric* OR pediatric* OR infant* OR Adolescen* OR Teen* OR Teenager* OR Young } \\
\text { OR Youth* }\end{array}$ \\
\hline Fonte: elaborada pelas autoras, 2020. &
\end{tabular}


sentados unidos, impossibilitando a separação entre criança e adolescentes indígenas para utilizar nessa revisão. Ao final, foram incluídos 10 artigos, o qual compõe a base de análise dessa pesquisa (Figura 2).

Os estudos incluídos foram realizados exclusivamente no Brasil, sendo os estados de Mato Grosso do Sul ${ }^{(16-19)}$ e Rondônia ${ }^{(7,}$ ${ }^{20-22)}$ predominantes, com quatro artigos cada (Quadro 2).

Os anos de publicação variaram entre 2004 a 2019, com predomínio do ano de $2013^{(17,18,20)}$, seguido de $2010^{(19,22)}$ (Quadro 2).

Segundo a população de estudo, metade ateve-se a descrição da distribuição de casos por faixa etária e não houve aprofundamento nas questóes socioepidemiológicas e clínicas ${ }^{(7,16,17,21,23)}$. Alguns estudos compararam a população indígena e não indígena $^{(17,21,23)}$, outros especificaram as etnias, tais como a Suruí, Kaiwá, Guaraní e Terena ${ }^{(7,15,21)}$ (Quadro 2).

Em relação ao tipo de estudo, identificou-se um predomínio de estudos epidemiológicos, descritivos, retrospectivos, realizados por meio de fontes se- cundárias, tais como pelo Sistema de Informação de Agravos de Notificação (SINAN) ${ }^{(17,20,21,23,24)}$, prontuários do Distrito Sanitário Especial Indígena (DSEI/ MS) ${ }^{(18)}$, formulários elaborados pelos autores ${ }^{(16)}$, registros com duas fontes, no Sistema de Informação de Saúde Indígena (SIASI) e na Fundação Nacional do Índio (FUNAI) ${ }^{(7)}$, ou no SINAN e em prontuários do DSEI/MS ${ }^{(19)}$. Apenas um estudo foi do tipo primário, realizado por meio de pesquisa de campo ${ }^{(22)}$.

A categorização da faixa etária divergiu entre os estudos, dependendo do objetivo investigado, variando entre menores de 1 ano a $20 \operatorname{anos}^{(7,16-21,23,24)}$. Ainda, a maioria dos estudos não relacionaram os achados com as diversas faixas etárias infanto-juvenil, limitando-se a relatar os dados apenas como crianças e/ ou adolescentes ou ainda com faixas etárias muito abrangentes, sem considerar os parâmetros e conceitos estabelecidos pela Organização Mundial da Saúde e/ ou do Ministério da Saúde.

O sexo masculino mostrou-se prevalente $^{(17,19,21,23,24)}$. Relativo à faixa etária

\section{Figura 2. Fluxograma das etapas de seleção dos artigos desta revisão} integrativa da literatura, Porto Velho, Rondônia, Brasil, 2021.
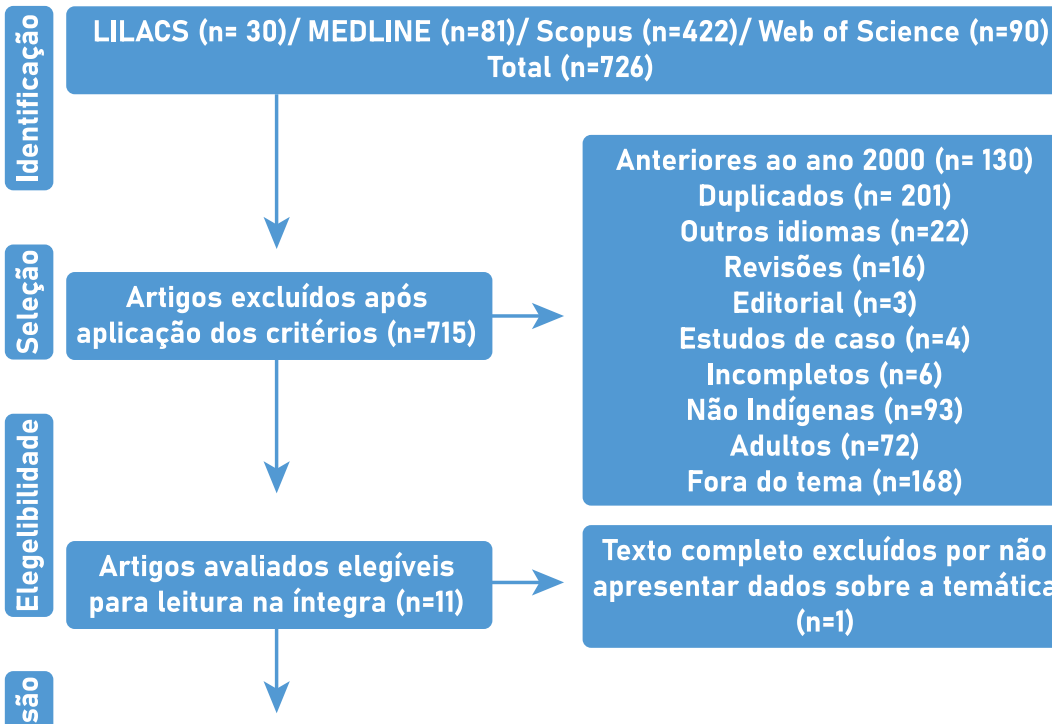

Texto completo excluídos por não apresentar dados sobre a temática $(n=1)$

\section{Artigos incluidos em síntese $(n=10)$}

de crianças indígenas com $\mathrm{TB}$, mais da metade dos estudos revelaram uma maior quantidade de casos absolutos entre crianças menores de $5 \operatorname{anos}^{(7,18-20,23,24)}$. Foi apontado que conforme aumenta a idade entre as crianças indígenas, a incidência de TB diminui ${ }^{(19)}$.

A variável clínica mais explorada refere-se ao exame de radiografia de tórax ${ }^{(7,}$ $16,20,22,24)$, apontando que quase $1 / 4$ dos pacientes foram submetidos ao tratamento sem ao menos realizar este exame $e^{(7,20,24)}$. Evidenciou-se, ainda, que a maioria dos casos entre crianças apresentavam apenas um tipo de alteração $^{(16)}$ relativo à classificação dos achados, cujos infiltrados e calcificações são os mais frequentes nessa população ${ }^{(22)}$.

$\mathrm{O}$ teste tuberculínico não foi realizado em mais de $80 \%$ dos $\operatorname{casos}^{(17,20,24)}$ ou não tiveram registros sobre a sua aplicação ${ }^{(7)}$.

Os parâmetros relativos às condiçóes nutricionais dos indivíduos, como a verificação do Índice de Massa Corpórea (IMC) e estado nutricional, que são necessários para o diagnóstico não laboratorial da criança, estavam presentes em apenas dois estudos ${ }^{(18,22)}$.

A forma clínica predominante foi a pulmonar $^{(7,17,19-21,23,24)}$, todavia foram encontradas formas extrapulmonares com maior expressão na ordem ganglionar, pleural, miliar, meníngea, osteomuscular, cutânea, laríngea, geniturinária e ocular ${ }^{(19,24)}$.

Foi apontado maior proporção de abandono do tratamento entre as crianças indígenas coinfectadas com o HIV e apenas um caso de TB drogaresistente ${ }^{(24)}$.

Quanto aos desfechos do tratamento, identificou-se elevada taxa de cura variando entre 86,8 a $91,1 \%{ }^{(19,20,24)}$. O abandono oscilou entre $3,6 \%$ e $7,2 \%$ e o óbito ficou entre 2,2\% e 4,6\% ${ }^{(19,24)}$ (Quadro 2).

\section{DISCUSSÃO}

Este estudo permitiu a identificação do perfil de saúde das crianças e adolescentes indígenas com $\mathrm{TB}$, a partir da literatura realizada exclusivamente no Brasil, o que mostra o interesse dessa temática 


\section{artigo}

Souza, G.A.S.C.; Ferreira, M.R.L.; Bonfim, R.O.; Orfão, N.H.;

Perfil de saúde da tuberculose entre crianças e adolescentes indígenas: uma revisão integrativa

Quadro 2. Síntese dos artigos incluídos nesta revisão, de acordo com o autor, ano, objetivo, delineamento, cenário, população e principais resultados dos estudos, Porto Velho, Rondônia, Brasil, 2021.

\begin{tabular}{|c|c|c|c|c|c|}
\hline $\begin{array}{l}\text { AUTOR, } \\
\text { ANO }\end{array}$ & $\begin{array}{l}\text { OBJETIVO DO } \\
\text { ESTUDO }\end{array}$ & $\begin{array}{l}\text { DELINEA- } \\
\text { MENTO DO } \\
\text { ESTUDO }\end{array}$ & $\begin{array}{l}\text { CENÁRIO } \\
\text { DO ESTUDO }\end{array}$ & POPULAÇÃO & PRINCIPAIS RESULTADOS \\
\hline $\begin{array}{l}\text { Basta et } \\
\text { al., } 2004\end{array}$ & $\begin{array}{l}\text { Caracterizar o } \\
\text { perfil epidemio- } \\
\text { lógico da TB no } \\
\text { grupo indígena } \\
\text { Suruí em Cacoal, } \\
\text { Rondônia. }\end{array}$ & $\begin{array}{l}\text { Epide- } \\
\text { miológico, } \\
\text { descritivo }\end{array}$ & Rondônia & $\begin{array}{l}\text { População } \\
\text { indígena } \\
\text { geral }\end{array}$ & $\begin{array}{l}\text { O estudo apontou que entre os indígenas, } 45 \% \text { dos } \\
\text { casos eram menores de } 15 \text { anos e } 1 / 4 \text { desse total eram } \\
\text { menores de cinco anos. Sendo que } 9 \% \text { dos casos } \\
\text { diagnosticados em crianças menores de } 10 \text { anos foram } \\
\text { realizados com baciloscopia. Ademais, o melhor índice } \\
\text { de casos confirmados microscopicamente foi na faixa } \\
\text { de } 15-20 \text { anos ( } 85 \% \text { ). Relativo às diferenças entre os } \\
\text { sexos foram pronunciadas nas faixas etárias de } 5 \text { - } 10 \\
\text { anos ( } 73.7) \text { e } 15 \text { - } 20 \text { anos }(90,9 \% \text { ) no sexo masculi- } \\
\text { no. Ainda se verificou que } 91 \% \text { das crianças realizaram } \\
\text { radiografia diagnóstica. }\end{array}$ \\
\hline $\begin{array}{l}\text { Basta et } \\
\text { al., } 2010\end{array}$ & $\begin{array}{c}\text { Descrever as } \\
\text { características } \\
\text { clinico- } \\
\text { radiológicas } \\
\text { em crianças e } \\
\text { adolescentes } \\
\text { identificados } \\
\text { como contatos } \\
\text { de doentes } \\
\text { de TB, aplicar } \\
\text { o sistema de } \\
\text { pontuação para } \\
\text { o diagnóstico de } \\
\text { TB na infância } \\
\text { e verificar se } \\
\text { as condutas } \\
\text { adotadas no } \\
\text { nível local foram } \\
\text { concordantes } \\
\text { com as diretrizes } \\
\text { nacionais. }\end{array}$ & $\begin{array}{c}\text { Clinico-epi- } \\
\text { demiológico, } \\
\text { descritivo }\end{array}$ & Rondônia & $\begin{array}{c}\text { Crianças } \\
\text { indígenas (< } \\
15 \text { anos) }\end{array}$ & $\begin{array}{l}\text { A média de idade foi de } 11,3 \text { anos, predomínio do sexo } \\
\text { feminino }(81,8 \%) \text {. Houve notificação em menores de } 15 \\
\text { anos em aproximadamente } 50 \% \text { dos casos. Relativo à } \\
\text { radiografia de tórax a maioria apresentou acometendo } \\
\text { do terço superior do pulmão. Foi realizado } 2 \text { ou mais } \\
\text { radiografias em } 27 \% \text { dos casos, e foram achados } 48,1 \% \\
\text { normais e } 51,9 \% \text { anormais, das quais } 22,2 \% \text { eram TB } \\
\text { provavelmente ativa e } 33,3 \% \text { sequelas. As alterações } \\
\text { restantes ( } 44,5 \% \text { ) foram consideradas sugestivas } \\
\text { de outras doenças. Houve predomínio de infiltrados } \\
\text { (38,9\%) e calcificações ( } 38,9 \% \text { ) e observou-se cavitações } \\
(11,1 \%) \text {, atelectasias/ derrame pleural ( } 11,1 \%) \text {. Sem } \\
\text { diferença no padrão radiológico entre indígenas e não } \\
\text { indígenas. O estudo mostrou crianças tratadas para } \\
\text { TB com esquema I (RHZ) e tratadas para ILTB com } \\
\text { isoniazida (H) apenas, o que discorda com as diretrizes } \\
\text { em } 52,6 \% \text { dos casos. Detectou-se déficit nutricional em } \\
21,1 \% \text { dos casos e observou-se a revacinação com BCG } \\
\text { intradérmico verificando-se a cicatriz vacinal. }\end{array}$ \\
\hline $\begin{array}{l}\text { Marques } \\
\text { et al., } 2010\end{array}$ & $\begin{array}{c}\text { Descrever } \\
\text { os aspectos } \\
\text { epidemiológicos } \\
\text { da tuberculose } \\
\text { na população } \\
\text { indígena com } \\
\text { idade inferior a } \\
\text { quinze anos, de } \\
\text { Mato Grosso do } \\
\text { Sul, Brasil, no } \\
\text { período de } 2000 \\
\text { a 2006, após a } \\
\text { implantação do } \\
\text { Subsistema de } \\
\text { Saúde Indígena. }\end{array}$ & $\begin{array}{c}\text { Epide- } \\
\text { miológico, } \\
\text { descritivo e } \\
\text { retrospectivo }\end{array}$ & $\begin{array}{l}\text { Mato Gros- } \\
\text { so do Sul }\end{array}$ & $\begin{array}{c}\text { Crianças } \\
\text { indígenas (< } \\
15 \text { anos) }\end{array}$ & $\begin{array}{l}\text { Predomínio em indivíduos com idade inferior a } 5 \text { anos } \\
\text { e elevado número de casos com idade inferior a } 1 \text { ano, } \\
\text { maior quantidade de caso do sexo masculino ( } 50,9 \% \text { ). A } \\
\text { proporção de casos de tuberculose em indígenas com } \\
\text { idade inferior a } 15 \text { anos foi de } 20,4 \% \text { ( } 224 / 1.096) \text {. Verifi- } \\
\text { cou-se elevados coeficientes de incidência de tuber- } \\
\text { culose em indígenas com menos de } 15 \text { anos de idade, } \\
\text { porém com curva descendente e uma queda anual em } \\
\text { torno de } 14 \% \text {. A forma pulmonar ( } 92,9 \% \text { foi a predomi- } \\
\text { nante, enquanto a distribuição das formas extrapulmo- } \\
\text { nares foi de } 7,1 \% \text {, sendo a ganglionar periférica de } 31,2 \% \\
(5 / 16) \text {, a pleural } 25 \% \text { ( } 4 / 16) \text {, a meningoencefalite } 18,8 \% \\
\text { (3/16), a miliar } 12,5 \% \text { (2/16) e a osteoarticular } 12,5 \% \\
\text { (2/16). Relativo ao desfecho } 91,1 \% \text { evoluíram para cura, } \\
2,2 \% \text { óbito } 3,6 \% \text { abandono do tratamento. A incidência } \\
\text { média de TB foi > 2.500/100.000 hab., no período de } \\
1991-2002 \text {. }\end{array}$ \\
\hline
\end{tabular}




\begin{tabular}{|c|c|c|c|c|c|}
\hline $\begin{array}{l}\text { Orellana et } \\
\text { al., } 2012\end{array}$ & $\begin{array}{c}\text { Analisar as ca- } \\
\text { racterísticas so- } \\
\text { ciodemográficas } \\
\text { e os indicadores } \\
\text { operacionais re- } \\
\text { ferentes ao con־- } \\
\text { trole da tubercu- } \\
\text { lose, comparando } \\
\text { indígenas e não } \\
\text { indígenas, no } \\
\text { estado de Rondô- } \\
\text { nia, no período de } \\
1997 \text { a } 2006\end{array}$ & $\begin{array}{c}\text { Epide- } \\
\text { miológico, } \\
\text { descritivo e } \\
\text { retrospectivo }\end{array}$ & Rondônia & $\begin{array}{c}\text { População } \\
\text { indígena e } \\
\text { não indígena }\end{array}$ & $\begin{array}{l}\text { Entre as notificações de indígenas 36\% dos casos eram } \\
\text { menores } 15 \text { anos. }\end{array}$ \\
\hline $\begin{array}{l}\text { Basta et } \\
\text { al., } 2013\end{array}$ & $\begin{array}{l}\text { Analisar caracte- } \\
\text { rísticas socio- } \\
\text { demográficas e } \\
\text { clínico epide- } \\
\text { miológicas dos } \\
\text { casos de tuber- } \\
\text { culose e fatores } \\
\text { associados ao } \\
\text { abandono e ao } \\
\text { óbito na vigência } \\
\text { do tratamento }\end{array}$ & $\begin{array}{l}\text { Epide- } \\
\text { miológico } \\
\text { descritivo } \\
\text { e analítico, } \\
\text { retrospecti- } \\
\text { vo, de base } \\
\text { populacional }\end{array}$ & $\begin{array}{l}\text { Mato Gros- } \\
\text { so do Sul }\end{array}$ & $\begin{array}{l}\text { População } \\
\text { indígena e } \\
\text { não indígena }\end{array}$ & $\begin{array}{l}\text { Maior quantidade de casos no sexo masculino, noti- } \\
\text { ficou-se } 13,5 \% \text { de indígenas em menores de } 10 \text { anos, } \\
\text { enquanto nas outras categorias raça/cor o adoecimento } \\
\text { em crianças não extrapolou } 5,0 \% \text {. A chance de aban- } \\
\text { dono foi duas vezes maior na raça/cor preta e parda } \\
\text { quando comparada aos indígenas de todas as idades. }\end{array}$ \\
\hline $\begin{array}{l}\text { Gava et al., } \\
2013\end{array}$ & $\begin{array}{c}\text { Avaliar os as- } \\
\text { pectos epide- } \\
\text { miológicos da } \\
\text { tuberculose em } \\
\text { crianças indíge- } \\
\text { nas brasileiras } \\
\text { e as ações para } \\
\text { seu controle. }\end{array}$ & $\begin{array}{c}\text { Epide- } \\
\text { miológico, } \\
\text { descritivo }\end{array}$ & Rondônia & $\begin{array}{l}\text { Crianças } \\
\text { indígenas e } \\
\text { não indíge- } \\
\text { nas (0-14 } \\
\text { anos) }\end{array}$ & $\begin{array}{l}\text { Predomínio do sexo masculino }(52,8 \%) \text {, notificação de } \\
60,8 \% \text { casos entre }<5 \text { anos, e } 84,8 \% \text { eram originários da } \\
\text { zona rural do estado. } 94,7 \% \text { dos casos indígenas tinhamam } \\
\text { a forma clínica pulmonar, a forma mista da TB predomi- } \\
\text { nou entre a faixa etária de } 10 \text { a } 14 \text { anos. As crianças, na } \\
\text { faixa etária de cinco a nove anos, foram acometidas por } \\
\text { TB extrapulmonar. Relativo aos exames diagnósticos, } \\
\text { a utilização de testes diagnósticos foi menor entre as } \\
\text { crianças indígena. Não foram realizados registros de } \\
85,7 \% \text { dos exame histopatológico em crianças, também } \\
\text { não foram realizados para confirmação diagnóstica em } \\
\text { crianças indígenas de zero a quatro anos, utilizou-se em } \\
\text { apenas } 10,0 \% \text { dos pacientes, a cultura foi realizada em } \\
\text { (2,6\%) dos casos, todavia, nenhum registro dos resul- } \\
\text { tados estava disponível para consulta, e a baciloscopia } \\
\text { foi realizada em } 26,4 \% \text { das notificações; radiografias de } \\
\text { tórax apresentaram resultados sugestivos de TB com } \\
\text { maior frequência na faixa etária de zero a quatro anos } \\
\text { (p }=0,047) \text {. Não houveram casos de coinfecção com } \\
\text { HIV em indígenas. A detecção dos casos foi classificada } \\
\text { como insuficiente e/ou regular em mais de } 80,0 \% \text { das } \\
\text { notificações entre indígenas, mostrando que a maioria } \\
\text { dos diagnósticos foi baseada na radiografia de tórax. } \\
\text { A adesão ao tratamento maior entre os indígenas. E a } \\
\text { incidência média foi maior entre os indígenas }<5 \text { anos, } \\
\text { no de } 2001 \text { (1.047,9 casos } / 100.000 \text { habitante). }\end{array}$ \\
\hline
\end{tabular}




\section{artigo}

\begin{tabular}{|c|c|c|c|c|c|}
\hline $\begin{array}{l}\text { Santos et } \\
\text { al., } 2013\end{array}$ & $\begin{array}{c}\text { Avaliar o } \\
\text { processo } \\
\text { diagnóstico } \\
\text { da tuberculo- } \\
\text { se pulmonar } \\
\text { em indígenas } \\
\text { menores } \\
\text { de } 15 \text { anos, } \\
\text { por meio do } \\
\text { Sistema de } \\
\text { Pontuação } \\
\text { do Ministério } \\
\text { da Saúde } \\
\text { Modificado } \\
\text { (SP-MSm), } \\
\text { em crianças e } \\
\text { adolescentes } \\
\text { com resulta- } \\
\text { dos negativos } \\
\text { na bacilos- } \\
\text { copia }\end{array}$ & $\begin{array}{l}\text { Epide- } \\
\text { miológico, } \\
\text { descritivo e } \\
\text { retrospectivo }\end{array}$ & $\begin{array}{l}\text { Mato Gros- } \\
\text { so do Sul }\end{array}$ & $\begin{array}{c}\text { Crianças } \\
\text { indígenas (< } \\
15 \text { anos) }\end{array}$ & 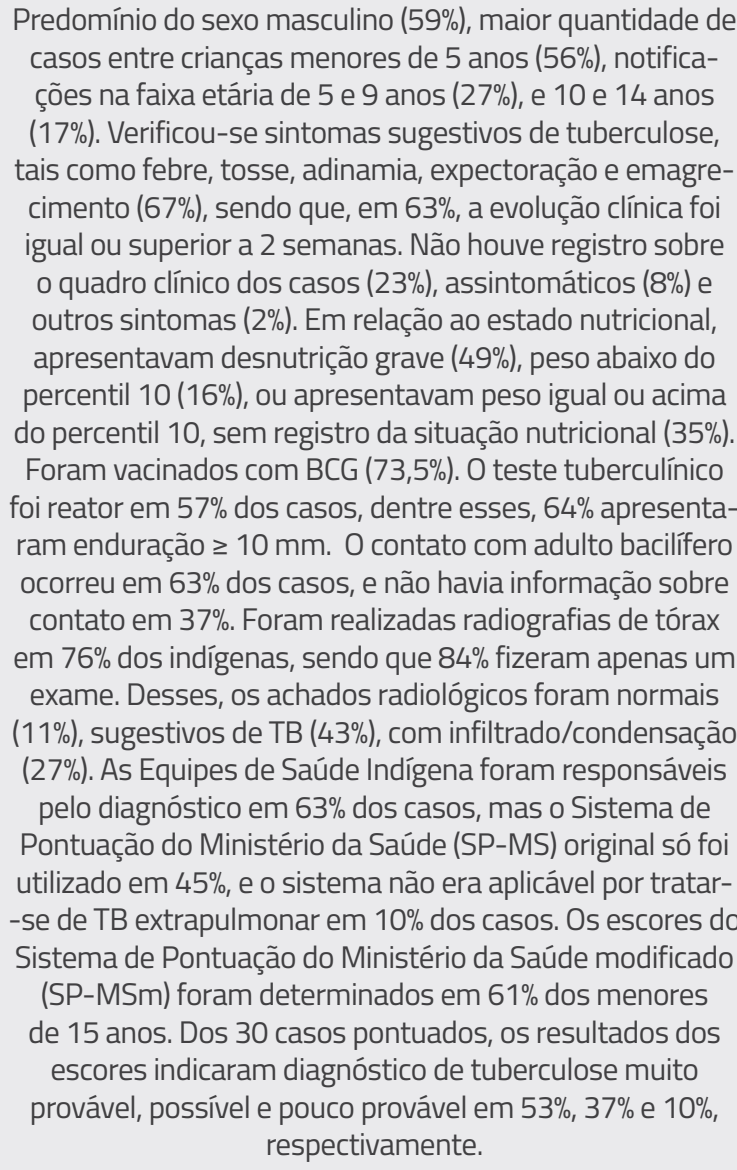 \\
\hline $\begin{array}{l}\text { Lachi \& } \\
\text { Nakaya- } \\
\text { ma, } 2015\end{array}$ & $\begin{array}{c}\text { Descrever } \\
\text { os aspectos } \\
\text { radiológicos } \\
\text { da tuberculo- } \\
\text { se pulmonar } \\
\text { em pacientes } \\
\text { indígenas } \\
\text { da cidade de } \\
\text { Dourados, } \\
\text { MS, Brasil, de } \\
\text { acordo com } \\
\text { idade e sexo. }\end{array}$ & $\begin{array}{l}\text { Epide- } \\
\text { miológico, } \\
\text { descritivo, } \\
\text { quantitativo } \\
\text { e retrospec- } \\
\text { tivo }\end{array}$ & $\begin{array}{l}\text { Mato Gros- } \\
\text { so do Sul }\end{array}$ & $\begin{array}{l}\text { População } \\
\text { indígena } \\
\text { geral }\end{array}$ & $\begin{array}{l}\text { O estudo apresentou } 8,6 \% \text { dos casos em menores de } 10 \\
\text { anos e } 8,6 \% \text { em adolescentes de } 10 \text { a } 19 \text { anos. Apontou, } \\
\text { ainda, a frequência de apenas um tipo de achado radiológi- } \\
\text { co em crianças com idade menor que } 10 \text { anos }(71,4 \%) \text { e em } \\
\text { adolescentes de } 10 \text { a } 19 \text { anos ( } 14,3 \%) \text {. Nenhuma criança } \\
\text { apresentou acometimento linfonodal ou atelectasia. Não } \\
\text { houve diferença estatisticamente significante entre as } \\
\text { faixas etárias - crianças } \leq 9 \text { anos, adolescentes de } 10 \text { a } 19 \\
\text { anos - nem na frequência dos diferentes tipos de achados } \\
\text { nas radiografias, na frequência de envolvimento de dife- } \\
\text { rentes áreas pulmonares ou na frequência de radiografias } \\
\text { anormais. Relativo ao teste de HIV a maioria das crianças } \\
\text { ( } 5 / 7 \text { ou } 71,4 \% \text { ) e adolescentes ( } 4 / 7 \text { ou } 57,1 \% \text { ) não realizou } \\
\text { e para os que realizaram, a sorologia para HIV foi negativa. }\end{array}$ \\
\hline $\begin{array}{l}\text { Mendes et } \\
\text { al., } 2016\end{array}$ & $\begin{array}{c}\text { Analisar a si- } \\
\text { tuação epide- } \\
\text { miológica da } \\
\text { tuberculose } \\
\text { no Rio Grande } \\
\text { do Sul, com } \\
\text { enfoque na } \\
\text { população } \\
\text { indígena }\end{array}$ & $\begin{array}{l}\text { Epide- } \\
\text { miológico, } \\
\text { descritivo }\end{array}$ & $\begin{array}{l}\text { Rio Grande } \\
\text { do Sul }\end{array}$ & $\begin{array}{l}\text { População } \\
\text { indígena } \\
\text { geral }\end{array}$ & $\begin{array}{l}\text { As crianças menores de } 10 \text { anos apresentaram } 12 \% \text { das } \\
\text { notificações da TB entre os indígenas e houve registro de } \\
\text { casos de tuberculose multirresistente (TBMR) entre povos } \\
\text { indígenas do Rio Grande do Sul no período. }\end{array}$ \\
\hline
\end{tabular}




\begin{tabular}{|c|c|c|c|c|c|}
\hline $\begin{array}{l}\text { Viana et } \\
\text { al., } 2019\end{array}$ & 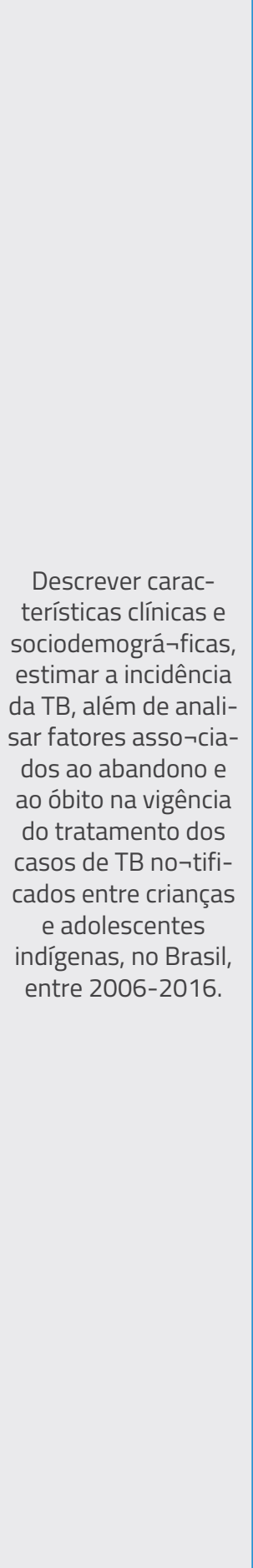 & $\begin{array}{c}\text { Epide- } \\
\text { miológico, } \\
\text { descritivo }\end{array}$ & Brasil & $\begin{array}{c}\text { Crianças e } \\
\text { adolescen- } \\
\text { tes indíge- } \\
\text { nas }\end{array}$ & 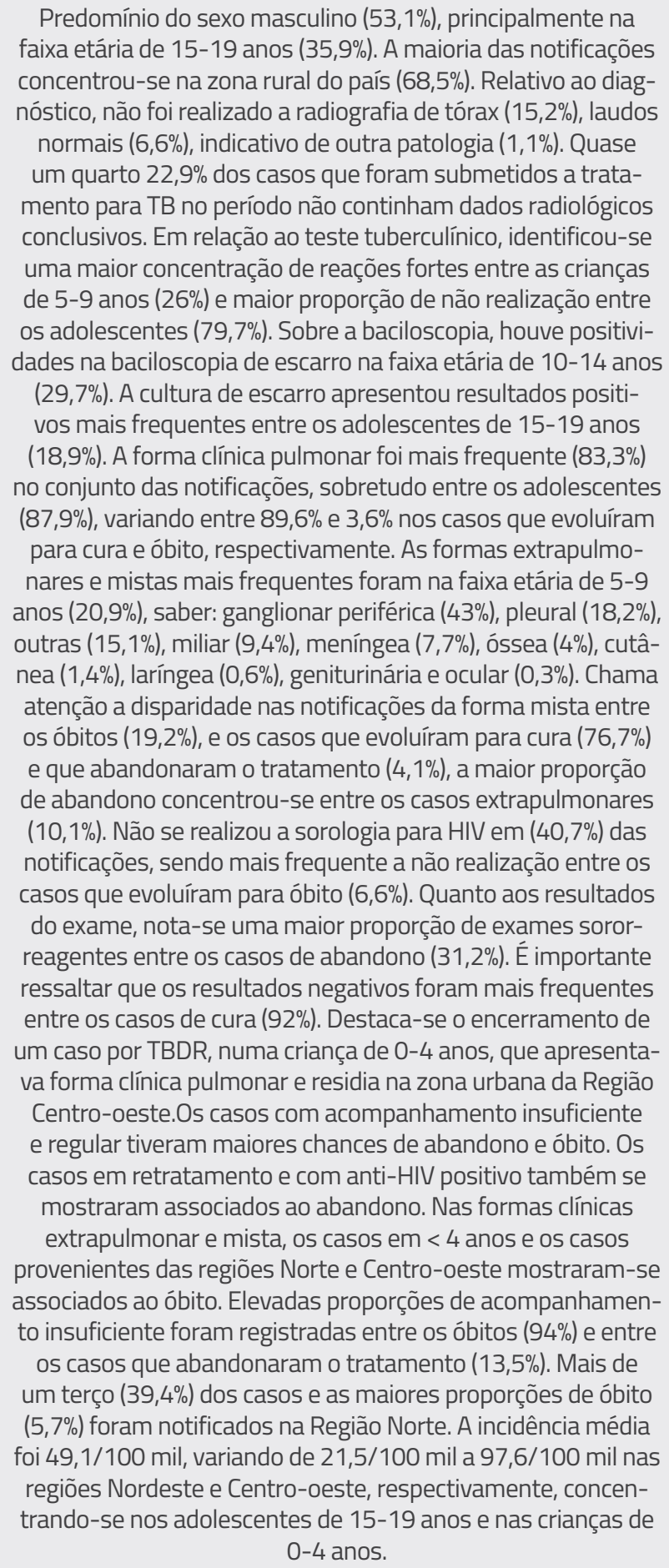 \\
\hline
\end{tabular}

no país, principalmente nos estados em que apresentam um maior quantitativo de indígenas ${ }^{(7,16-22)}$.
Por outro lado, identificou-se uma lacuna de estudos com recorte de faixa etária entre indígenas em outros países.
Todavia, estudos que tenham essa configuração são necessários, pois permitem a descrição das subjetividades no processo 
saúde-doença para a população indígena, relacionando-os aos fatores nutricionais ${ }^{(18,19,22)}$, imunológicos ${ }^{(17)}$, socioculturais $^{(22)}$, organizacionais ${ }^{(7,21)}$, bem como as desigualdades regionais ${ }^{(23,24)}$, que modificam o perfil epidemiológico para que ocorra uma distribuição equitativa dos recursos $^{(17)}$, acesso universal com ênfase na promoção, proteção e recuperação da saúde ${ }^{(24)}$, treinamento de pessoal ${ }^{(17)} \mathrm{e}$ a prevenção dos agravos nas aldeias ${ }^{(21)}$, a fim de garantir maior equidade.

Evidenciou-se uma heterogeneidade quanto aos dados epidemiológicos e clínicos dessa população ${ }^{(7,17-21,23,24)}$, na medida em que não havia padronização referente a faixa etária. Todavia, estudos descritivos com as crianças indígenas de diversas faixas etárias identificam e caracterizam a proporção de crianças infectadas, que são casos sentinelas, e por meio delas é possível compreender e monitorar os casos de infecção recente entre os adultos e auxiliar na implantação de medidas e controles voltados para a realidade dessa população ${ }^{(19,20)}$. Dessa forma, é possível avaliar o desempenho do Programa Nacional de Controle da TB (PNCT) relativo à realização da vigilância desses casos pela Equipe de Saúde Indígena ${ }^{(7)}$.

$\mathrm{O}$ acesso ao banco de dados específicos para a população indígena o SIASI deve ser estimulado, pois houve dificuldade na realização de estudos que propuseram descrever e correlacionar os achados epidemiológicos, clínicos e desfechos com as etnias. Os autores tiveram que associar o sobrenome ao povo, uma vez que é costume de a população ter a etnia como sobrenome ou ainda, cruzar os dados do endereço do paciente com georreferenciamento das aldeias ${ }^{(20,21)}$.

A radiografia de tórax (RX) e o teste tuberculínico (TT) não foi realizado em todos os casos suspeitos ${ }^{(7,18,20-24)}$, como recomenda o Ministério da Saúde para crianças e adolescentes com baciloscopia negativa ou Teste Rápido Molecular para a TB (TMR-TB) não detectado. Uma justificativa relativa ao número reduzido de RX deve-se as distâncias entre a localização do aparelho em relação as aldeias, uma vez que os exames são realizados mediante parcerias do DSEI e as Secretarias Municipais de Saúde. Podem, ainda, haver dificuldades na interpretação radiológica da imagem pela falta de emissão de laudo, o que desestimula a solicitação do exame ${ }^{(18)}$, além do acesso restrito aos cuidados em saúde no emprego dos exames diagnósti$\cos ^{(24)}$. Todavia, a realização do $\mathrm{RX}$ é rápida, tem baixo custo e é fundamental para agilizar o diagnóstico ${ }^{(16)}$.

O RX e o TT são exames complementares a avaliação clínica, e fazem parte do sistema de pontuação do Ministério da Saúde que está sendo realizado com baixa aplicabilidade para o diagnóstico da TB infanto-juvenil ${ }^{(18,20,21)}$, cujo público frequentemente não apresenta confirmação bacteriológica ${ }^{(18)}$. Ademais, houve constatação de discordâncias entre o diagnóstico e o sistema de pontuação, uma vez que o tratamento foi realizado sem elementos suficientes para confirmação da TB, foi realizado tratamento para ILTB em paciente que deveria ter recebido quimioterapia para TB, bem como em pacientes que deveriam ter sido tratados para ILTB e não recebeu qualquer terapêutica ${ }^{(22)}$.

A avaliação do estado nutricional, bem como a investigação de anemias e parasitoses intestinais foi realizada em poucos estudos, entretanto ela é necessária para compreender os fatores que favorecem a transmissão da $\mathrm{TB}$, visto que a desnutrição compromete a resposta imunológica, e as aldeias apresentavam precárias condições socioeconômicas $^{(22)}$. Ainda, foi verificado elevadas taxas de desnutrição nos anos de 2003 e 2004 na região da Grande Dourados, em Mato Grosso do Sul, que poderia estar associado ao aumento de TB na região e período supracitado ${ }^{(19)}$.

O desfecho de cura nas crianças e adolescentes indígenas foi maior do que a população em geral ${ }^{(19,20,24)}$ e maior do que a recomendação da Organização Mundial da Saúde (OMS) que é, no mínimo, de $85 \%^{(1)}$. Isso pode ser reflexo do acompanhamento dos casos durante o tratamento após a criação do subsistema de Atenção à Saúde Indígena, melhor efetividade da estratégia do tratamento supervisio- nado da $\mathrm{TB}^{(19)}$ e/ ou viés de seleção no tocante ao diagnóstico, visto que os casos registrados podem ser os casos que são acompanhados e tiveram maior acesso ao serviço de saúde ${ }^{(23)}$.

Ademais, nota-se que o abandono e óbito são maiores do que o recomendado pela OMS de, no máximo, $5 \%{ }^{(1)}$. Esses dados estão associados ao acompanhamento inadequado dos casos e dificuldades para realização do diagnóstico precoce em crianças de 0-4 anos, relacionando-se as dificuldades regionais e há expressiva diferença entre as faixas etárias ${ }^{(24)}$. De modo complementar, podem ter relação ao ineficaz desempenho do PNCT e do subsistema de saúde indígena, além da incapacidade de vigilância dos casos nos serviços de saúde por causa das disparidades no acesso ao diagnóstico e acompanhamento até o término do tratamento ${ }^{(7,17,21,23)}$.

Estudo apontam sobre a importância de investimento em ações concretas para reduzir a pobreza e desigualdade para haver melhoria na incidência $\mathrm{da} T \mathrm{~TB}^{(17)}$, bem como na atenção à saúde indígena, no que diz respeito a conformidade da detecção dos casos de TB com prioridade para as recomendações nacionais e internacionais ${ }^{(20,23)}$.

\section{CONCLUSÕES}

O perfil de saúde da TB entre crianças e adolescentes indígenas evidenciou a caracterização da população sem descrição étnica, com maior incidência da forma pulmonar, sexo masculino e em crianças menores de 5 anos. As dificuldades permeiam a não sistematização da busca dos achados clínicos, não documentação das condições nutricionais e a baixa realização de exames de apoio diagnóstico, tais como a RX e TT para a investigação da TB infanto-juvenil.

Evidencia-se, portanto, a necessidade de ações intersetoriais, educação permanente em saúde para os profissionais atuantes, considerando especialmente a transculturalidade, desenvolvimento de ações de vigilância e a articulação entre os serviços, bem como a ampliação do 
acesso aos serviços de saúde e exames diagnósticos.
A limitação desse estudo está relacionada à busca exclusiva em bases de dados indexadas, o que faz com que estudos publicados em revistas não indexadas não fossem utilizadas.

\section{REFERÊNCIAS}

1. World Health Organization. Global Tuberculosis Report. [Internet]. Geneva. 2020. Available from: . https://apps.who.int/iris/ bitstream/handle/10665/336069/9789240013131-eng.pdf

2. Brasil. Manual de recomendações para o controle da tuberculose no Brasil. [Internet]. Brasília. 2019. Available from: http://www.aids.gov.br/pt-br/pub/2019/manual-de-recomendacoes-para-o-controle-da-tuberculose-no-brasil

3. United Nations. Political Declaration of the UN General Assembly High-level meeting: End TB United Nations High-Level Meeting on the fight against tuberculosis 26 September 2018. 2018. https://www.who.int/tb/unhlmonTBDeclaration.pdf

4. Australian Governement. The indigenous Australians' health Programme. Departamento of health. [Internet]. Australian 2018. Available from: https://www1.health.gov.au/internet/main/publishing.nsf/Content/indigenous-programme-Ip\#: :text=The\%20 Indigenous\%20Australians $\% 20$ Health\%20Programme,-The\%20Australian\%20Government\&text=The\%20objective\%20of\%20the $\% 20$ IAHP,and\%2Oremote\%20locations\%20across\%20Australia.

5. National Collaborating Centre for Aboriginal Health (NCCAH). The Aboriginal Health Legislation and Policy framework in Canada. [Internet]. Canadá. 2011. Available from: http://www.arnbccommunitiesofpractice.ca/ahnn/wp-content/uploads/2015/03/ Health-Legislation-Policy-eng.pdf

6. Brasil. Fundação nacional de Saúde. Política de Atenção à Saúde dos Povos Indígenas. [Internet]. Brasilia. 2002. Available from: https:// bvsms.saude.gov.br/bvs/publicacoes/politica_saude_indigena.pdf

7. Basta PC, Junior CEAC, Escobar AL, Santos RV. Epidemiologic aspects of tuberculosis in the Suruí Indians, Brazilian Amazon. Rev. Soc. Bras. Med. Trop. 2004; 37(4): 338 342. https://doi. org/10.1590/S0037-86822004000400010

8. Malacarne J, Gava C, Escobar AC, Santos R S, Basta PC. Health service access for tuberculosis diagnosis and treatment among Indigenous Peoples in Rondônia state, Brazilian Amazon, 2009-2011: a cross-sectional study. Epidemio. Serv. Saúde. 2019; 28(3): e2018231. https://doi.org/10.5123/s167949742019000300002

9. Rao VG, Bhat J, Yadav R, Sharma RK, Muniyandi M. A comparative study of the socio-economic risk factors for pulmonary tuberculosis in the Saharia tribe of Madhya Pradesh, India. Transactions of the Royal Society of tropical medicine and Hygiene. 2018; 112(6): 272278. https://doi.org/10.1093/trstmh/try052

10. Faust L, McCarthy A, Schreiber Y. Recommendations for the screening of paediatric latent tuberculosis infection in indigenous communities: a systematic review of screening strategies among higth-risk groups in low-incidence countries. BMC Public Health. 2018; 18 (1): https://doi.org/10.1186/s12889-018-5886-7

11. Deslile M, Seguin J, Zeilinshi D, Moore DL. Peadiatric abdominal tuberculosis in developed countries: case series and literature review. Archives of Disease in Children. 2016;101(3):253-258. https://doi.org/10.1136/archdischild-2015-308720
12. Botelho LLR, Cunha CCA, Macedo M. O método da revisão Integrativa nos estudos organizacionais. Rev. Gestão e Sociedade. 2011; 5(11):121-136. https://doi.org/10.21171/ges.v5i11.1220

13. Ouzzani M, Hammady H, Fedorowicz Z, \& Elmagarmid A. Rayyan - a web and mobile app for systematic reviews. Systematic Reviews.2016; 5 (210): https://doi.org/10.1186/s13643-016-0384-4

14. Landis JR, Koch GG. The measurement of observer agreement for categorical data. Biometrics. 1977; 33(1):159-174. https:/doi. org/10.2307/2529310

15. Moher D, Liberati A, Tetzlaff J, et al. Preferred Reporting Items for Systematic Reviewsand MetaAnalyses: The PRISMA Statement. PLoS Med 2009; 6(7):e1000097.https:/dx.doi.org/10.1371/journal.pmed.1000097

16. Lachi A, Nakayama M. Radiological findings of pulmonary tuberculosis in indigenous patients in Dourados, MS, Brazil. Radiol. Bras. 2015; 48(5): 275-281. http:/dx.doi.org/10.1590/0100-3984.2014.0070

17. Basta PC, Marques M, Oliveira RL, Cunha EAT, Resendes APC, Santos RS. Social inequalities and tuberculosis: an analysis by race/ color in Mato Grosso do Sul, Brazil. Rev. Saúde Pública. 2013; 47(5): 854-864; https://doi.org/10.1590/S0034-8910.2013047004628

18. Santos SC, Marques AMC, Oliveira R L, Cunha RV. Scoring system for the diagnosis of tuberculosis in indigenous children and adolescentes under 15 years of age in the state of Mato Grosso do Sul, Brazil. J. bras. pneumol. 2013; 39(1): 84-91. https://doi.org/10.1590/S180637132013000100012

19. Marques AMC, Pompilio MA, Santos SC, Garnês SJA, Cunha RV. Tuberculosis among Brazilian indigenous individuals aged less than 15 yearsold in State of Mato Grosso do Sul, Brazil. Rev. Soc. Bras. Med. Trop. 2010; 43(6): 700-704. https:/doi.org/10.1590/50037-86822010000600020

20. Gava C, Malacarne J, Rios DPG, SantAnna CC, Camacho LB, Basta PC. Tuberculosis in indigenous children in the Brazilian Amazon. Revista de Saúde Pública. 2013; 47(1): 77-85. https://doi. org/10.1590/S0034-89102013000100011

21. Orellana JDY, Gonçalves MJF, Basta PC. Sociodemographic features and operating indicators of tuberculosis control between indigenous and non-indigenous people of Rondônia, Western Amazon, Brazil. Rev. bras. Epidemiol. 2012; 15(4): 714-724. http:// dx.doi.org/10.1590/S1415-790X2012000400004

22. Basta PC, Rios DPG, Alves LCC, Sant`Anna CC, Junior CEAC. Clinical and radiological study of Suruí indigenous children and adolescents, Amazon Region, Brazil. Rev. Soc. Bras. Med. Trop. 2010; 43(6): 719-722. http://dx.doi.org/10.1590/S0037-86822010000600024

23. Mendes AM, Bastos JL, Bresan D, Leite MS. Epidemiologic situation of tuberculosis in Rio Grande do Sul: an analysis about Sinan's data between 2003 and 2012 facusing on indigenous peoples. Rev. bras. Epidemiol. 2016; 19(3): 658-669. https:/doi.org/10.1590/1980-5497201600030015

24. Viana PVS, Cadenotti SB, Bierrenbach AL, Basta PC. Tuberculosis in Indigenous Children and adolescentes in Brazil: factors associated with death and tratamento dropout. Cad. Saúde Pública. 2019; 35(3): e0007421. https://doi.org/10.1590/0102-311X00074218 